\title{
CCD PHOTOMETRY OF THE ECLIPSING BINARY HV 2274 IN THE LARGE MAGELLANIC CLOUD
}

\author{
R.D. WATSON, S.R.D. WEST, WILLIAM TOBIN \& A.C. GILMORE \\ Mount John University Observatory \\ Department of Physics, University of Canterbury, Christchurch \\ New Zealand
}

\begin{abstract}
B, V$ and $I_{c}$ photometry of the eclipsing binary HV 2274, located in the LMC, was obtained with the CCD system at the Mount John University Observatory, Lake Tekapo, New Zealand during 1990 . Over 100 data points were acquired in each filter to an estimated precision of $0.02 \mathrm{mag}$. The flat maxima evident in the light curves suggest that the system, which is currently classed as an interacting candidate, should probably be considered as a detached one. A revised orbital period based on the inclusion of current data is presented. Eclipse timings indicate an eccentric orbit. An apsidal period in the vicinity of $120 \mathrm{yr}$ is likely.
\end{abstract}

\section{Introduction}

The periodic nature of HV 2274 [R.A. $4^{\mathrm{h}} 39^{\mathrm{m}}$ Dec. $-68^{\circ} 25^{\mathrm{m}}(1990), \mathrm{m}_{p g} \sim 13.8$ ] was first identified by Leavitt (1908). Shapley and McKibben (1942) appreciated its binary nature and classed it as a $\beta$ Lyrae type. Shapley and Nail (1953) removed previous doubts about the system's membership of the LMC and revised the earlier ephemeris by determining more accurate photographic light elements. Gaposhkin (1970) presented a photographic light curve of the system, Payne-Gaposchkin (1971) improved upon the 1953 ephemeris, and Gaposhkin (1977) lists times of minima. Wood et al. (1980) included the system in their finding list of interacting binaries.

We have obtained a CCD light curve of HV 2274, with some 330 data points in $B$, $V$ and $I_{\mathrm{c}}$. These observations were acquired at the Mount John University Observatory (MJUO), Lake Tekapo, New Zealand from April 1990 to November 1990. The observations were part of MJUO's ongoing commitment to long-term CCD photometry of variable stars in the Magellanic Clouds. At a southerly latitude of $44^{\circ}$, MJUO is well suited to year-round monitoring of these objects.

\section{Instrumental System and Observations}

Observations were made on a 0.6 metre Boller \& Chivens equatorially-mounted Cassegrain reflector. A Photometrics PM3000 CCD system, incorporating an overcoated Thomson TH7882 CDA chip, was used as the detector. The chip has $384 \times 576$ pixels, each $23 \mu \mathrm{m}$ square. Each pixel corresponded to an angular size of 0.6 arcsec on the Boller \& Chivens. The filters, consisting of Schott glass, were made to a recipe specified by Dr. M. S. Bessel 
of Mount Stromlo and Siding Springs Observatories to give a system response close to standard.

For flat-fielding, the telescope was focussed to infinity and pointed at a illuminated white board mounted on the inside of the dome at the start of a night's observations. Because we had no facility for guiding, our exposures of HV 2274 were kept to a maximum 400 seconds. Our experience was that a longer exposure time, particularly at a higher airmass, could lead to a marked degree of image elongation in declination. The seeing measured in our images ranged from $3.0 \pm 0.7 \operatorname{arcsec}$ in $B$ to $2.5 \pm 0.5 \operatorname{arcsec}$ in $I$. Such seeing values are typical of the maritime climate of New Zealand and its geographic position in the Roaring Forties. Two stars were selected as comparisons for HV 2274. In the process of determining the best comparison stars in the field, CCD magnitudes were obtained for ten other stars within the field. None of these were found to be variable over the time baseline of our observations and to the precision of the photometry.

\section{Data Reduction}

Because of the nature of the HV 2274 field, it was prudent to use a crowded-field photometric reduction package. We employed the RomAFOT package (Buonanno et al. 1983) which is incorporated into the European Southern Observatory's Munich Image and Data Analysis System (Grosbøl 1989). ROMAFOT iteratively fits the sum of analytically determined point spread functions to the signal level in any selected small window of an image, while simultaneously accounting for sky background. This background may have the form of an inclined plane. The interactive facilities and the quality of the image display provided by ROMAFOT were found to be of great value in the reduction process. For our HV 2274 reductions we adopted a uniformly weighted Moffat function (Moffat 1969) as the representative point spread function.

A number of systematic effects commonly appear in our reduced CCD data. One such effect results from imperfect flat-fielding. This means that there are often low spatialfrequency errors, at the $1-2 \%$ level, remaining in our results. This effect does not matter if the star images are registered at similar locations on the CCD chip each time. However, because of telescope orientation, we have the field in some images rotated by $180^{\circ}$ compared to the field in other images. This often requires a correction factor between the results for the two orientations, which we determine by choosing a check star close to the variable, and calculating the mean magnitude offset between the two field orientations for the check comparison. This magnitude offset may then be applied to the variable - comparison result. Such a corrective term was necessary for the HV 2274 observations reported here.

Another systematic effect sometimes present is due to a second-order extinction term. This gives an airmass-dependent correction in some cases where there is a marked colour difference between the variable or check and the mean comparison. Despite having selected one very blue and one red comparison here, we found no need to apply an airmass related correction term to the present data. Similarly we sometimes see systematic effects related to poor seeing, but find no significant evidence requiring a seeing correction term in the HV 2274 data.

\section{Results and Discussion}

Figure 1 presents our $B, V$ and $I_{\mathrm{c}}$ light curves for HV 2274. Based on the data for check stars examined, the standard deviation of our eclipsing variable data is estimated to be about 0.02 magnitudes. Observations on one night were obtained through the deepest points of 


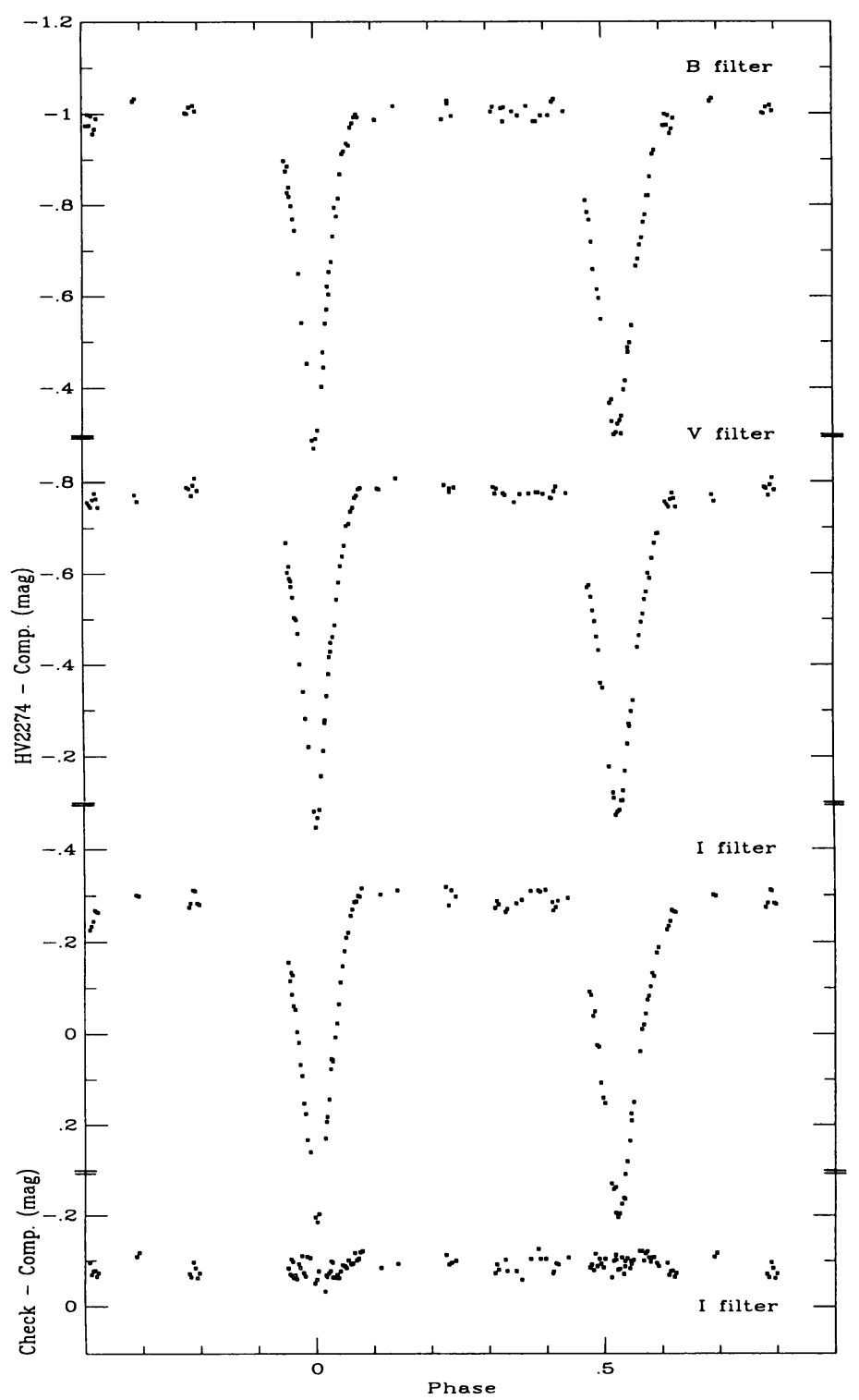

Figure 1. Lightcurves for HV 2274 in each of the $B, V$ and $I_{\mathrm{c}}$ filters. The primary minimum has been corrected by an observed $0.0860 \pm 0.0004$ phase offset when compared to the predictions of the Payne-Gaposchkin (1971) ephemeris. The ordinate is the instrumental magnitude of the variable minus the instrumental magnitude of the mean intensity of the two chosen comparison stars. The results for a check of comparable signal strength in the $\mathrm{I}_{c}$ filter, are shown for comparison. 
the primary minimum. Observations on another night similarly covered the deepest points of the secondary minimum. Observed times of primary minimum occurred late relative to the zero of phase determined by the Payne-Gaposchkin (1971) ephemeris. The average phase offset for the three filters was $+0.0860 \pm 0.0004$, as measured by parabolic leastsquares fitting of the light curves near primary minimum. It is also clear from the light curves that the primary and secondary minima are not equally spaced, suggesting an orbital eccentricity. Least squares fitting of secondary minima give a phase of $0.524 \pm 0.001$ relative to the primary minima. We have used the results of our fitting of primary and secondary minima to establish the accurate epochs given in Table 1. Then, combining the mean of these epochs with Gaposhkin's (1971) published times of minima (excluding two highly deviant points), we derived a revised period of $P=5.72601 \pm 0.00004 d$.

TABLE 1. HV 2274 Light Curve Data

\begin{tabular}{|c|c|}
\hline \multicolumn{2}{|c|}{$\begin{array}{c}\mathrm{E}_{\mathrm{o}}(\mathrm{prim})=2448099.818 \pm 0.002 \mathrm{HJD} \\
\mathrm{E}_{\mathrm{o}}(\mathrm{sec})=2448102.817 \pm 0.004 \mathrm{HJD} \\
\mathrm{P}=5.72601 \pm 0.00004 \mathrm{~d} \\
\text { Secondary eclipse phase }=0.524 \pm 0.001\end{array}$} \\
\hline Primary eclipse depths & $\begin{aligned} \Delta \mathrm{m}_{B} & =0.72 \\
\Delta \mathrm{m}_{V} & =0.71 \\
\Delta \mathrm{m}_{I_{\mathrm{c}}} & =0.70\end{aligned}$ \\
\hline Secondary eclipse depths & $\begin{aligned} \Delta \mathrm{m}_{B} & =0.70 \\
\Delta \mathrm{m}_{V} & =0.70 \\
\Delta \mathrm{m}_{I_{\mathrm{c}}} & =0.69\end{aligned}$ \\
\hline
\end{tabular}

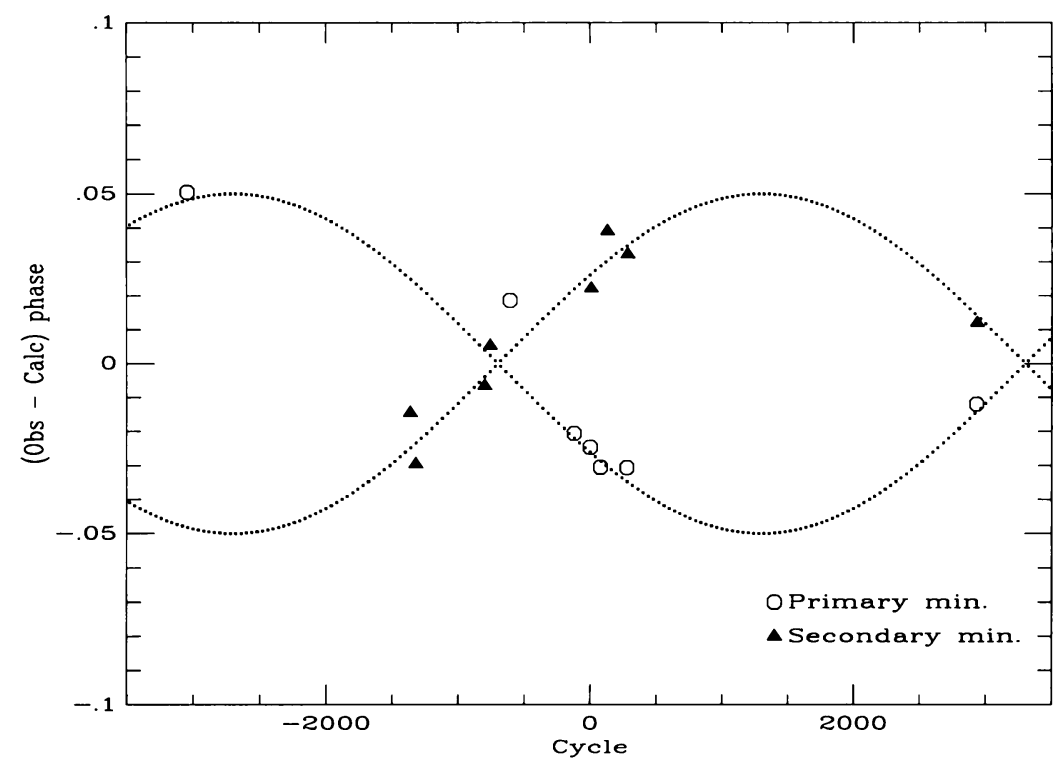

Figure 2. Phase residuals of primary and secondary minima in HV 2274 based on the ephemeris presented here. The data here is consistent with an apsidal period of about 8000 cycles (120 years), which is shown by the schematically drawn sinusoids. 
The depth of primary and secondary minima are only marginally different, with values of $\Delta m=0.70 \pm 0.02$ applying to data from all filters. Primary eclipses occur over a phase interval of approximately 0.15 , and secondary eclipses last about 30 percent longer. To the precision of our data, flux appears constant outside of the eclipse regions. Hence our data suggests a system containing two detached B stars moving in an elliptical orbit observed nearly edge on. We will presently use the Wilson \& Devinney code to quantify the constraints that the light curve places on the system's properties. One further interesting facet of the eclipse timing data is seen in Figure 2, where residuals from the present ephemeris are shown separately for primary and secondary minima, again excluding the two highly deviant points in the Gaposhkin (1977) list. The data, though sparse, is clearly evocative of an apsidal period in the vicinity of 8000 cycles, or about $120 \mathrm{yr}$.

\section{References}

Buonanno, R., Buscema, C., Corsi, E., Ferraro, I., \& Iannicola, G., (1983) Astr. Astrophys., 126, 278.

Gaposhkin, S. I., (1970) Smithsonian Astrophysical Observatory Special Report, 310.

Gaposhkin, S. I., (1977) Smithsonian Astrophysical Observatory Special Report, 380.

Grosbøl, P., (1989) Reviews in Modern Astronomy, ed Krane, G., 2, p. 242.

Leavitt, H. S., (1908) Annals of Harvard College Observatory, 60, 87.

Moffat, A.F.J., (1969.) Astr. Astrophys., 3, 455.

Payne-Gaposchkin, C. H., (1971) Smithsonian Contributions to Astrophysics, 13.

Shapley, H., McKibben, V., (1942) Harv. Bull., 916, 19.

Shapley, H., Nail, V. McKibben, (1953) Proceedings of the National Academy of Sciences, U.S. A., 39, 1 .

Wood, F. B., Oliver, J. P., Florkowski, D. R., Koch, R. H., (1980) Finding List for Observers of Interacting Binary Stars, $5^{\text {th }}$ edition, University of Pennsylvania Press. 